\title{
Participación laboral de las mujeres rurales chilenas: tendencias, perfiles y factores predictores*
}

\author{
Carlos Rodríguez Garcés** \\ Johana Muñoz Soto*** \\ Recibido: 2014-10-06 Aprobado: 2015-02-02 Disponible en línea: 2015-15-03 \\ doi:Io.III44/Javeriana.cdrı2-75.plmc
}

Cómo citar este artículo: Rodríguez, C., \& Muñoz, J. (2015). Participación laboral de las mujeres rurales chilenas: tendencias, perfiles y factores predictores. Cuadernos de Desarrollo Rural, I2(75), 77-98. http://dx.doi.org/10.III44/ Javeriana.cdri2-75.plmc

\footnotetext{
* Este artículo forma parte y es un producto del proyecto de investigación Reconfiguraciones socioespaciales, estructurales y simbólicas de la población, los modos de vida, el trabajo y la familia en el marco de las nuevas relaciones ruralurbanas (Código II40579), financiado por el Fondecyt.

** Doctor por la Universidad de Barcelona. Catedrático en la Universidad del Bío-Bío, encargado de área del Centro de Investigación y Desarrollo en Informática Educativa (Cidcie) y miembro del grupo investigador de Desarrollo Local Sostenible. Correo electrónico: carlosro@ubiobio.cl *** Investigadora Ayudante del Centro de Investigación en Informática Educativa (Cidcie), de la Universidad del Bío-Bío. Correo electrónico: jmunoz@alumnos.ubiobio.cl
} 


\title{
Resumen
}

Mediante un Modelo de Regresión Logística se analiza la participación laboral de las mujeres rurales y los factores asociados. Se observa un aumento en la tasa de participación con reducidos niveles de desempleo. No obstante, persisten brechas y precarización de las condiciones de trabajo. Las mujeres con mejor preparación y con actitudes más favorables hacia el trabajo generan ingresos, sostienen a su familia y desarrollan procesos de empoderamiento. Los factores que favorecen la probabilidad de estar empleada son: número perceptores de ingreso y de hijos, ser jefa de hogar, el nivel de escolaridad y la edad. Por otra parte, los factores inhibidores son: tener hijos menores o en edad preescolar, tener pareja y disponer de un ingreso mayor no dependiente del aporte femenino.

\section{Palabras clave:}

participación laboral; mercado del trabajo; mujer rural; trabajo remunerado

\section{Labor Participation of Chilean Rural Women: Tendencies, Profiles and Predicting Factors}

\begin{abstract}
We analyze the labor participation of rural women and the associated factors by means of Logistic Regression Model. We observe an increse in the participation rates, with reduced unemployement levels, despite the persistance of gaps and precariousness in work conditions. Women with a better preparation and with more favorable attitudes towards work conditions generate income, support their families, develop empowerment processes. The following improve the probability to be employed: number of income receivers and children; being the head of household; level of education; age. Inhibiting factors are: having children who are minors or are in pre-school age; having a partner; and having a higher income not dependent of female contribution.
\end{abstract}

\section{Keywords:}

labor participation; labor market; rural women; paid work 


\section{Participation rural de femmes rurales chiliennes: tendances, profiles et facteurs prédicteurs}

\section{Résumé}

Au moyen d'un modèle de régression logistique on analyse la participation au travail des femmes rurales et les facteurs associés. On observe un augmente dans le taux de participation avec de bas niveaux de chômage, néanmoins il y a encore brèches et précarisation des conditions du travail. Les femmes avec la meilleur préparation et avec les attitudes les plus favorables envers le travail produisent les revenus, elles entretient la famille et developpent des processus de leaders. Cela contribue à la probablité d'être employée : le numero de percepteurs de revenus et des fils et filles ; être chef de la maison, scolarité, âge. Les facteurs inhibiteurs sont : avoir de petits enfants ou des enfants en âge scolaire, être en couple et avoir une plus grand revenu non dependant d'une contribution femenine.

Mots clés:

participation au travail; marché du travail; femme rurale; travail récompensé

\section{Participação laboral das mulheres rurais chilenas: tendências, perfis e fatores preditores}

\section{Resumo}

Mediante Modelo de Regressão Logística foi analisada a participação laboral das mulheres rurais e os fatores associados. Observa-se um aumento na taxa de participação com reduzidos níveis de desemprego. No entanto, continuam a existir fendas e precarização das condições de trabalho. As mulheres com maior nível de instrução e com atitudes mais favoráveis frente ao trabalho geram rendas, sustêm a família e desenvolvem processos de empoderamento. Os fatores que favorecem a probabilidade de estar empregada são: número recebedores de renda e de filhos, ser chefa da família, nível de escolaridade e idade. De outro lado, os fatores inibidores são: ter filhos menores ou em idade pré-escolar, ter casal e dispor de uma renda maior não dependente da contribuição feminina.

\section{Palavras-chave:}

participação laboral; mercado do trabalho; mulher rural; trabalho remunerado 


\section{Introducción}

Desde 1990 se han constatado cambios profundos en la sociedad y en la economía rural chilena. Se inicia un acelerado proceso de asalarización de la fuerza de trabajo, el mercado laboral se diversifica y se hace menos dependiente de la rama silvoagropecuaria como fuente de ingresos y generadora de empleos; además, la mujer irrumpe con fuerza en la escena económica nacional.

El aumento de la participación laboral de las mujeres ha ido acompañado de los procesos de urbanización, diferenciación y mercantilización de las economías mundiales (Weller, 1998), tendencia con potencial de crecimiento, en especial, en países como Chile dada su manifestación tardía y exclusión histórica de la mujer del mercado del trabajo, aun considerando las diferentes estructuras etarias, educacionales y niveles de ingreso (Contreras y Plaza, 2007), que tienden a ser más acentuadas en el campo.

Las remuneraciones de la mujer por la realización de un trabajo mejora la distribución del ingreso y se constituye en poderosa herramienta para superar situaciones de pobreza y facilita el acceso a mayores niveles de bienestar económico, individual y familiar, posibilitado por el consumo de bienes y servicios en variedad y calidad (Bravo, Contreras y Puentes, 2005; Contreras y Plaza, 2010). Al diversificarse los perceptores de renta asociados con el trabajo disminuye la probabilidad del hogar de caer bajo la línea de pobreza, y lo hace menos dependiente de las transferencias del Estado (Bonvecchi y Smulovitz, 2008). Una mayor participación laboral repercute en los presupuestos nacionales y hace aumentar la masa de cotizantes al sistema previsional, lo que da mayor viabilidad a emprendimientos en materia de seguridad social, hecho relevante en un país como Chile que envejece con rapidez (Bravo y Bertranou, 2006).

En el ámbito social visibiliza a las mujeres como eficientes prestadoras de servicios y generadoras de productos con rentabilidad; en lo privado las habilita como agentes económicos por medio de las transferencias monetarias que estas hacen al hogar (Fawaz y Rodríguez, 2013). Situación que, especialmente en sectores rurales, la valida en el aspecto social y ante su grupo familiar, pues refuerza su autonomía, rompe su aislamiento y amplía sus horizontes emocionales y cognitivos (Anthopoulou, 2010); esto genera mecanismos de empoderamiento y poder de negociación al interior de la familia (Lamanna y Klasen, 2008), incluso su capacidad de sobrevivir fuera de esta (Agarwal, 1999), lo que constituye un eficiente mecanismo para reducir las desigualdades de género en lo social y económico (De Ferranti et al., 2005). Como contraparte, al disponer exclusivamente del aporte de sus parejas, se sienten con pocos 
derechos a decidir sobre usos alternativos de estos ingresos que no sea el cubrir las necesidades básicas familiares (Benería, 1987; Haugen y Blekesaune, 2005).

No obstante la importancia que tiene la participación laboral femenina, esta solo ha sido estudiada recientemente en Chile y con preferencia desde modelos de capital humano (Contreras y Plaza, 2007). En general, estos estudios muestran que la probabilidad de participar en el mercado del trabajo disminuye conforme aumentan los costos relativos asociados con la constitución de familia y tenencia de hijos/as (Attanasio, Low y Sanchez-Marcos, 2004), en especial, en mujeres en edad reproductiva, con hijos/as pequeños y menor capital humano acumulado (Bravo, Contreras y Puentes, 2005). Incluso, en las mujeres con mayor escolaridad, el efecto negativo que registra la presencia de hijos/as en la participacion laboral tiende a atenuarse o desaparecer (Cruces y Galiani, 2007), dada la valoracion que hace el mercado del trabajo respecto de las credenciales educativas. En esta misma linea, la existencia de redes de cuidado infantil y sus costos son determinantes en la oferta laboral femenina (Connelly, 1992; Fong y Lokshin, 2000; Gelbach, 2002; Blau y Currie, 2006; Jaumotte, 2003; Berlinsk, Galiani, y Manacorda, 2007) sobre todo en aquellos sectores más vulnerables de la población (Fong y Lokshin, 2000; Cascio, 2006).

El salario potencial del mercado tiene efectos en la decisión de ejercer una actividad remunerada por parte de las mujeres (Harbart, Muchnik, Strüver y Vial, I99I), quienes demuestran mayor elasticidad a los cambios de precio de mercado del trabajo que sus pares los hombres (Henríquez, Mizala y Romaguera, 1999), por lo que se retrotrae al hogar cuando los costos de conciliar trabajo y familia hacen la actividad laboral escasamente rentable; esta es una consecuencia asociada también con el tiempo que demanda la realización de actividades de tipo doméstico para la mujer (Ballara, Damianović y Parada, 20I0). La respuesta al mercado laboral esta determinada por la relación entre los retornos potenciales del salario y la productividad de la madre en el hogar, la cual se acentua con la existencia de hijos/as pequeños que requieren cuidados constantes (García, Glinskaya y Lokshin, 2000), en especial, en madres con bajo nivel de escolaridad y en hogares donde el ingreso familiar proviene del trabajo de la pareja varón de manera significativa.

En sintonía con lo anterior, se observa que el mayor ingreso total del hogar afecta de forma negativa la probabilidad de que una mujer con pareja ingrese al mercado laboral (Bordón, 2006). La presencia de pareja, situación que se manifiesta con mayor tendencia en mujeres con hijos/as, provoca incluso que se sobreestime el efecto de estos últimos sobre la participación laboral (Larrañaga, 2006). La importancia de la pareja varón, como fuente de ingresos del hogar y en la participación laboral 
femenina, está asociado tanto a la estructura del mercado del trabajo como a patrones de orden cultural.

El mercado del trabajo para las mujeres tiende a ser más restrictivo, concentrado en determinadas ocupaciones y áreas de la economía donde se les presentan menores barreras de entrada, situación que conduce a una precarización de los salarios (Meller, 2010). El componente cultural por su parte, configura una familia tradicional donde el hombre asume el rol de proveedor y la mujer el de cuidadora del hogar y de los hijos o hijas (Schkolnik, 2004); por tanto queda escindida del mercado laboral, limitada a las labores domésticas y en subordinación al varón (Valdés, 2007). Las caracteristicas culturales del medio expresado en el grado de machismo y predominancia de valores conservadores tienen fuerte influencia y contrarrestan en más del doble la incidencia del capital humano sobre la participación laboral (Contreras y Plaza, 2007).

En síntesis, en atención a las externalidades positivas que genera la participación laboral femenina en el entorno personal, familiar y social, así como la creciente visibilización e importancia que adquiere la mujer en el medio rural, como agente económico generador de ingresos y de sustento familiar (rol y reconocimiento que hasta el momento le ha sido esquivo), es que se hace necesario determinar las tendencias que sigue la participacion laboral femenina en sectores rurales, caracterizar el perfil sociolaboral de la mujer que trabaja, y establecer los factores que desincentivan u obtaculizan su incorporación en el mercado del trabajo.

\section{Metodología}

La presente investigación hace uso de la base de datos histórica de los censos poblacionales de los años 1982, 1992 y 2002 del Instituto Nacional de Estadística (INE), además de la Encuesta de Caracterización Socioeconómica Nacional (Casen) del año 20II. Con el fin de homologar esta encuesta con las bases censales, se le ponderó por factor de expansión regional de base Censo 2002. Con estas fuentes de datos secundarios se realiza un ánalisis longitudinal para describir la tendencia de la participación en el mercado del trabajo de las mujeres rurales, y se caracteriza su perfil sociolaboral en el horizonte temporal de los últimos 30 años. En forma especial, con la Casen 20 I se realiza un análisis de Regresión Logística Bivariante y se modela la opción dicótomica de la decisión de participar o no en el mercado del trabajo, mediante la identificacion del impacto o incidencia que en ella genera 
un conjunto de variables explicativas incorporadas al modelo, y que tendrían algún sustento según el análisis teorico previamente realizado.

Para ambos análisis se define Participación Laboral Femenina como la condición de encontrarse trabajando (Ocupadas) o en búsqueda de trabajo (Desocupadas). Variable de análisis que, para la condición de ocupada y en un nivel más desagregado, establece las categorías de trabajadoras asalariadas e independientes para la población de 15 años de edad y más, y aquellas que desarrollan una actividad para obtener beneficios o ganancia familiar como aprendices o en práctica. Resolución que también incluye Familiares No Remunerados, siempre que trabajen por lo menos una hora. Condición de Ocupada que se mantiene aún por ausencias temporales, constatadas al momento de la encuesta, sea por enfermedad, vacaciones, huelga o permiso. Asimismo, bajo la condición de Desocupada se considera a la población de 15 años de edad y más que durante la semana anterior a la realización de la encuesta se encontraban sin empleo, pero que en el último mes han realizado esfuerzos concretos para conseguirlo, por lo que prima este último componente como expresión de disposición efectiva para iniciar un trabajo en el futuro.

Mención especial precisa la categoría Familiar No Remunerado, la cual está subsumida dentro de la condición de Ocupada. Esto hace mención a la actividad productiva desarrollada por la mujer bajo administración de un familiar y sin recibir retribución en dinero o especies por ese trabajo. Habitualmente incluye mujeres que colaboran en la actividad del cónyuge, así como hijas que ayudan a sus padres; y si bien es una actividad que tiende a invisibilizar históricamente el trabajo de las mujeres en el campo, es una categoría de menor preponderancia al compararla con otras manifestaciones de la participación laboral, dado el proceso de asalarización que acontece en el mundo rural.

El modelo de Regresión Logística Binaria propuesto establece una variable dependiente de carácter dicotómica $\left(\mathrm{Y}_{\mathrm{it}}\right)$, dada por la decisión de participar, donde I define a la mujer rural que participa en el mercado laboral en el año t y o si es que no participa; además de un conjunto de variables explicativas. Se busca así el modelo que prediga de forma más exhaustiva la probabilidad de inserción laboral femenina con base en la presencia de un conjunto reducido de variables. El modelo estadístico está configurado mediante la siguiente ecuación:

\footnotetext{
$Y_{i t}=\beta 0+\beta_{1}$ Edad $+\beta_{2}$ Pareja $+\beta_{3}$ Escolaridad $+\beta_{4}$ Hijos $<5+\beta 5$ Hijos $5 y 15+\beta_{6}$ Hijos $>I$

$5+\beta_{7}$ Hijos $<16+\beta_{8}$ Ingr.familiardesc.ingresotrabajomujer $+\beta_{9}$ Mujerjefadehogar +

$\beta_{I 0}$ Perceptoresdeingresos $+\beta_{I I}$ Ingresofamiliar
} 
Donde:

Cuadro 1. Variables incluidas en el Modelo de Regresión Logística

\begin{tabular}{|c|c|}
\hline$Y_{i t}$ & $\begin{array}{l}\text { Variable dependiente dicotómica que consigna la participación laboral femenina, } \\
\text { mediante los valores de r y o (participación laboral o ausencia de esta) }\end{array}$ \\
\hline Edad & $\begin{array}{l}\text { Edad en años cumplidos, variable continua que fluctúa entre } 24 \text { años y } 60 \text { años } \\
\text { de edad, en mujeres, por considerarlas en edad económicamente activas }\end{array}$ \\
\hline Pareja & $\begin{array}{l}\text { Variable dicotómica expresada en la presencia (I) o ausencia (o) de pareja al interior } \\
\text { del hogar, con independencia de la naturaleza del vínculo (casada o conviviente) }\end{array}$ \\
\hline Escolaridad & $\begin{array}{l}\text { Años de escolaridad o capital humano. Variable continua } \\
\text { que reside entre los o años y } 22 \text { años de educación }\end{array}$ \\
\hline Hijos<5 & $\begin{array}{l}\text { Variable dicotómica que adopta valor I cuando hay presencia de } \\
\text { hijos menores de } 5 \text { años en el hogar y o cuando no los hay }\end{array}$ \\
\hline Hijossyls & $\begin{array}{l}\text { Variable dicotómica que adopta valor I cuando hay presencia de hijos en el } \\
\text { hogar con edades comprendidas entre los } 5 \text { y los I5 años y o cuando no lo hay }\end{array}$ \\
\hline Hijos $>15$ & $\begin{array}{l}\text { Variable dicotómica que adopta valor I cuando hay presencia de hijos } \\
\text { en el hogar con edades mayores de } 15 \text { años y o cuando no lo hay }\end{array}$ \\
\hline Hijos $<16$ & Variable continua que expresa la cantidad de hijos menores de i6 años en el hogar \\
\hline $\begin{array}{l}\text { Ingr.Familiar desc. } \\
\text { Ingreso Trabajo Mujer }\end{array}$ & $\begin{array}{l}\text { Variable continua que expresa el ingreso autonómo familiar, en miles } \\
\text { de pesos, una vez excluido el aporte económico que realiza la mujer } \\
\text { a este con ocasión de la realización de un trabajo remunerado }\end{array}$ \\
\hline Mujer Jefa de Hogar & $\begin{array}{l}\text { Variable dicotómica que explora la existencia del hogar } \\
\text { bajo jefatura femenina (I) o masculina (०) }\end{array}$ \\
\hline Perceptores de Ingresos & $\begin{array}{l}\text { Variable continua que expresa la cantidad de personas } \\
\text { que contribuyen económicamente al hogar }\end{array}$ \\
\hline Ingreso Familiar & Variable continua que expresa el ingreso autonómo familiar del hogar. \\
\hline
\end{tabular}

FuENTE: elaboración propia

Este modelo de Regresión Logística Binaria se expresa en un conjunto de índices de regresión parcial, donde el signo expresa la orientación o sentido del impacto del factor, y su valor numérico la magnitud o intensidad que tiene tal incidencia en la variable predicha. El modelo quedó constituido por i variables predictoras con un nivel de significancia en la prueba ómnibus de $\alpha<0.00$, informando que este ayuda a explicar el evento (participación laboral de las mujeres rurales). El modelo además presenta un alto poder explicativo con índices de variabilidad explicada, que oscilan entre 0,587 y un 0,793 según sea la prueba utilizada, $\mathrm{r}^{2}$ de Cox y Snell o $\mathrm{r}^{2}$ de Nagelkerke, respectivamente. Por último, el porcentaje global de clasificaciones que es capaz de predecir correctamente el modelo es de $92.2 \%$. En términos particulares clasifica correctamente a $85.2 \%$ 
de las mujeres rurales empleadas (especificidad) y a $96.9 \%$ de las mujeres no empleadas (sensibilidad), por tanto, se trata de un modelo de alta capacidad predictiva. El análisis estadístico descriptivo y multivariante fue realizado mediante el uso del sofware SPSS en su versión 2I.0.

\section{Análisis}

Cuadro 2. Participación laboral según sexo y zona, Chile ig82-20ir (\%)

\begin{tabular}{lllllll} 
& Urbano & \multicolumn{3}{c}{ Rural } & \multicolumn{3}{c}{ Variación } \\
\cline { 2 - 7 } & Hombre & Mujer & Hombre & Mujer & MR/MU & MR/HR \\
\hline Censo 1982 & 71.3 & 26.6 & $8 \mathrm{I} .4$ & II.4 & $-57 . \mathrm{I}$ & -86.0 \\
\hline Censo 1992 & 68.9 & 30.0 & 73.6 & 12.0 & -60.0 & -83.7 \\
\hline Censo 2002 & 70.5 & 37.8 & 67.3 & 19.0 & -49.7 & -71.8 \\
\hline Casen 20II & 70.2 & 45.2 & 69.7 & 30.8 & -31.9 & -55.8 \\
\hline
\end{tabular}

Nota: MR/MU es la variación expresada en porcentaje que evidencia la participación laboral de las mujeres rurales respecto de las mujeres urbanas. MR/HR es la variación expresada en porcentaje que evidencia la participación laboral de la mujer en el sector rural respecto de la participación del hombre del sector rural.

FuENTE: INE y Casen, años respectivos, elaboración propia

En el Cuadro 2 se muestran las tendencias y variaciones de la participación en el mercado del trabajo en Chile en los último 30 años, esto es, personas de I5 años de edad y más, que se encuentran trabajando (Ocupados) o buscando trabajo de manera activa (Desocupados), es básicamente masculina, no obstante evidenciarse un sostenido crecimiento de la tasa de participación de la mujer en el periodo analizado. La irrupción femenina, sobre todo en el sector rural, se constituye en uno de los elementos más significativos y transformadores del mercado del trabajo, a pesar de que Chile se caracterice por tener todavía una baja participación en este segmento en el contexto internacional. En el año 1982 tan solo II.4\% de las mujeres rurales señalaban estar laborando; en 201 r esta proporción se situó en $30.8 \%$, lo que representa un aumento de $170 \%$, en particular, durante el último decenio. En efecto, a partir de 2000 no solo aumenta la tasa de participación de las mujeres rurales que trabajan, sino que además se reducen las brechas tanto por zona y sexo de modo significativo.

Una constelación de factores explica este fenómeno. El desarrollo de actividades no agrícolas, muchas fuera del contexto rural; la movilidad temporal, 
facilitadas por las vías de comunicación e infraestructuras, son factores importantes. Se suma la incipiente feminización de determinadas actividades económicas, dada la diversificación y flexibilización del mercado laboral a la oferta femenina, alejadas de las actividades limitadas y tradicionales del pasado. Eventos que operan en concomitancia con el mayor interés de la mujer de elevar sus condiciones de vida y de acceder a diversidad de bienes y servicios (Farah y Pérez, 2004; Kay, 2008), posibilitado por el mayor capital humano disponible, y también por una revalorización de la autonomía, disposición actitudinal favorable frente al trabajo y al consumo (Fawaz y Rodríguez, 2013).

Aunque se observan diferencias en la actividad laboral femenina según la edad, el nivel de estudios y la situación familiar, entre otros, el creciente aumento de mujeres en la población activa no ha significado un incremento de la tasa de desocupadas. Los niveles de desempleo transitan entre $13 \%$ y $10 \%$ durante todo el periodo analizado. El mercado del trabajo muestra capacidad para absorber el aumento de mano de obra disponible, lo que se expresa en concordancia con la proliferación del empleo temporal. En otras palabras, la demanda de trabajo femenino se moviliza con base en las necesidades de mano de obra de la actividad económica, tanto rural como urbana, pero esta creación de nuevos empleos, frente al aumento de la oferta, actúa también en la disminución los salarios y precarización de condiciones laborales. En consecuencia, el trabajo femenino en el sector rural no solo se desvaloriza como trabajo productivo, sino que tiende a ser considerado como parte de la fuerza de trabajo asalariada temporal o vinculada al sector informal de la economía, asimilada a labores agrícolas, artesanía y comercio (Ballara, Damianović y Parada, 2010).

Es así como la modalidad de contrato estacional y flexible, expresión patente de inestabilidad laboral, tiene amplia presencia en el campo y con mayor fuerza entre las mujeres. En 20II, el empleo temporal y ocasional alcanzaba $36 \%$ en el sector rural y solo $17.6 \%$ en el sector urbano. Asimismo, dentro de zonas rurales esta cifra asciende a 39.3\% en el grupo de las mujeres (Casen, 20II). Es decir, una oferta de trabajo condicionada por la estacionalidad productiva y para un contingente de preferencia femenino y con reducidas capacidades para competir por posiciones más ventajosas. 


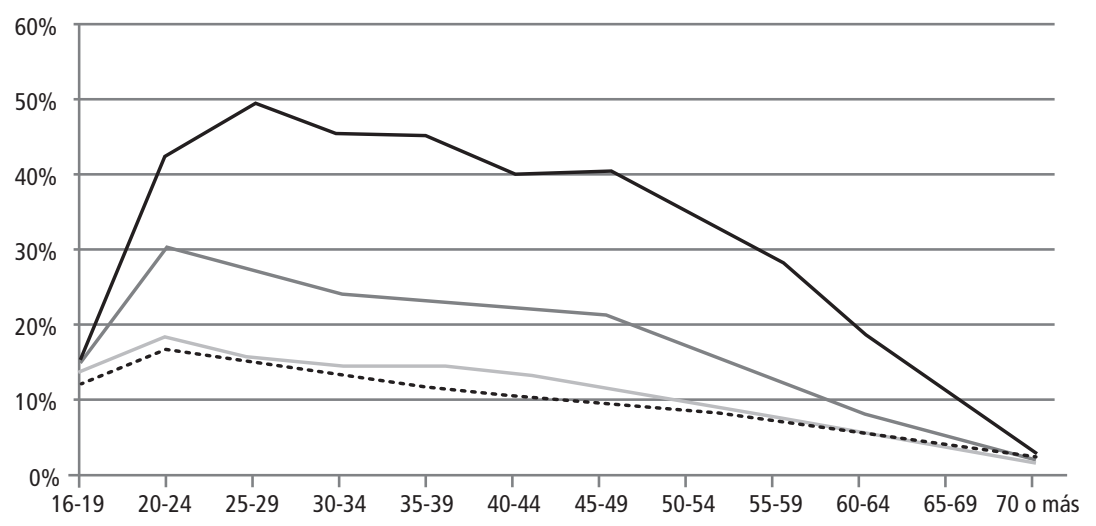

..... Censo 1982 - Censo 1992 - Censo 2002 - Casen 2011

Gráfico l. Tendencia de la participación laboral de las mujeres rurales según cohorte de edad

Fuente: Censo Casen, elaboración propia

El gráfico señala los perfiles de la participación femenina en zonas rurales según rango etario para los distintos periodos censales. Se observa la fuerte homogeneidad en el comportamiento de la participación en el periodo intercensal 1982-1992, solo con leves diferencias por rango etario. Perfiles que comienzan a diferenciarse a partir del año 2002, periodo que marca la mayor y constante irrupción de la mujer en la actividad económica, así como un comportamiento más diferenciado según el rango etario. A partir de esta fecha los parámetros de la tasa de participación femenina se constatan cada vez más estrechamente vinculados con la edad, pero en un patrón de relación de comportamiento cóncavo. Similar comportamiento evidencia la participación femenina en zonas urbanas según informan los hallazgos de Bravo, Contreras y Puentes (2005).

Que el punto modal de la inserción laboral sea, precisamente, el rango etario 25-29 años, estaría relacionado con la fase de finalización de estudios. Las generaciones más jóvenes extienden su permanencia en el sistema educativo con el fin de ingresar mejor equipadas al mercado laboral. Cuanto mayor es el nivel educacional de una mujer, mayor es su salario potencial y, asimismo, mayor es el coste de oportunidad de participar, lo que aumenta la probabilidad de hacerlo (Álvarez, 2002). El incremento de la participación laboral femenina en sectores rurales es también un efecto de generaciones mejor preparadas.

Del mismo modo, las pautas de comportamiento de las mujeres respecto del mercado laboral se relacionan con el estadio del ciclo vital y varían de manera 
considerable a lo largo de este. $\mathrm{Al}$ aumentar la edad o cambiar de cohorte, sea como resultado de ser un contingente menos preparado o consecuencia de la constitución de familia y llegada de los hijos, la mujer se retrotrae al hogar. Para el año 20II, en todos los rangos etarios, en especial, desde los 25 a los 54 años, una de cada tres mujeres rurales con pareja legal o de hecho integraban el mercado laboral, mientras que entre las mujeres separadas o sin pareja estas cifras superaban $55 \%$. Discrepancias asociadas, en el caso de mujeres separadas o con hijos/as, con las mayores responsabilidades que ellas asumen como agente generador de ingresos familiares.

$\mathrm{Al}$ disponer de pareja y ser este quien genera los ingresos, la mujer se retrotrae al hogar para cumplir con otras responsabilidades familiares (Castro, García y Badillo, 20II) y tareas domésticas, las cuales reducen su tiempo e inserción como fuerza laboral y desincentiva la inversión en capital humano (Becker, 1985). La decisión de participar en el mercado laboral depende de las preferencias o perfil actitudinal hacia el trabajo, así como de los ingresos no laborales disponibles, por lo que el salario del cónyuge se puede considerar como un ingreso no laboral cuando la mujer toma la decisión de trabajar (Bravo, Contreras y Puentes, 2005). Frente a la existencia en el hogar de ingresos no laborales percibidos como suficientes, se tiende a reducir la actividad laboral remunerada por parte de las mujeres, quienes se dedican con mayor intensidad al trabajo doméstico, reproductivo o productivo del hogar.

En efecto, las mujeres emparejadas no solo son menos activas en el mercado laboral y trabajan menos horas, sino que además salen de este al momento de entrar a una unión conyugal o cuando llegan los hijos; no obstante, tal comportamiento se ve atenuado como resultado de la acción del nivel educativo alcanzado y las condiciones del empleo, elementos que tienden a ser más favorables en los grupos más jóvenes y de mayores ingresos.

El diferencial educativo ejerce presión sobre la participación laboral entre los cónyuges en función de un efecto renta. Las mayores remuneraciones con ocasión de un mayor nivel educativo hacen menos necesarios los ingresos del otro (Castro, García y Badillo, 20II). Las mujeres rurales más jóvenes prolongan su permanencia escolar, retardan la nupcialidad y la llegada de los hijos, y se incorporan al mercado laboral no solo con mayor nivel de escolaridad que las generaciones que le precedieron sino que además lo hacen solteras, por tanto, presentan una menor inclinación a engrosar el sector inactivo al momento de constituir familia que antaño.

Así por ejemplo, en el año 201 en el grupo entre 20-24 años de edad de las mujeres rurales solo $6.6 \%$ estaba casada, $42.4 \%$ se encontraba ocupada o buscaba trabajo y, entre los 25-29 años, $24.8 \%$ estaba casada, $49.6 \%$ ocupada o buscando trabajo (Casen, 20II). En 
I982 en cambio, estas cifras eran diametralmente distintas. En el rango 20-24 años 43.5\% se encontraba casada y un escaso $17 \%$ integraba la fuerza de trabajo. Para el rango de 25-29 años, 65.2\% estaba casada y I5.2\% ocupada o buscando trabajo.

Las familias rurales ya no pueden prescindir de los ingresos aportados por el trabajo, y menos del generado por las mujeres. A medida que la actividad laboral y la generación de ingresos posibilita bienestar, consumo y desarrollo personal, subordina fuertemente la actividad reproductora, por lo que es lógico que decisiones racionales tendientes a maximizar la satisfacción de los primeros se encaminen a retardar y planificar la llegada de los hijos; esto reconfigura la estructura familiar y redefine, aunque no sin dificultad, los roles tradicionales de género instalados en el campo.

En la actualidad, las mujeres rurales, en especial, las más jóvenes y más educadas, conciben su desarrollo personal, reconocimiento social y logro del bienestar familiar ligado a la inserción en el mercado del trabajo, no obstante, esta participación se encuentra limitada por un conjunto de factores de relevancia sociocultural sobre todo en espacios territoriales marcados por la persistencia de tradicionalismos. Es así como se suman elementos asociados con la presencia de pareja, tenencia de hijos/as, en especial en edades menores, roles en la estructura familiar y niveles de ingresos, a los tradicionales factores de rango etario y nivel de capital humano.

Cuadro 3. Factores asociados con la participación laboral de las mujeres rurales Modelo de Regresión Logística Binaria

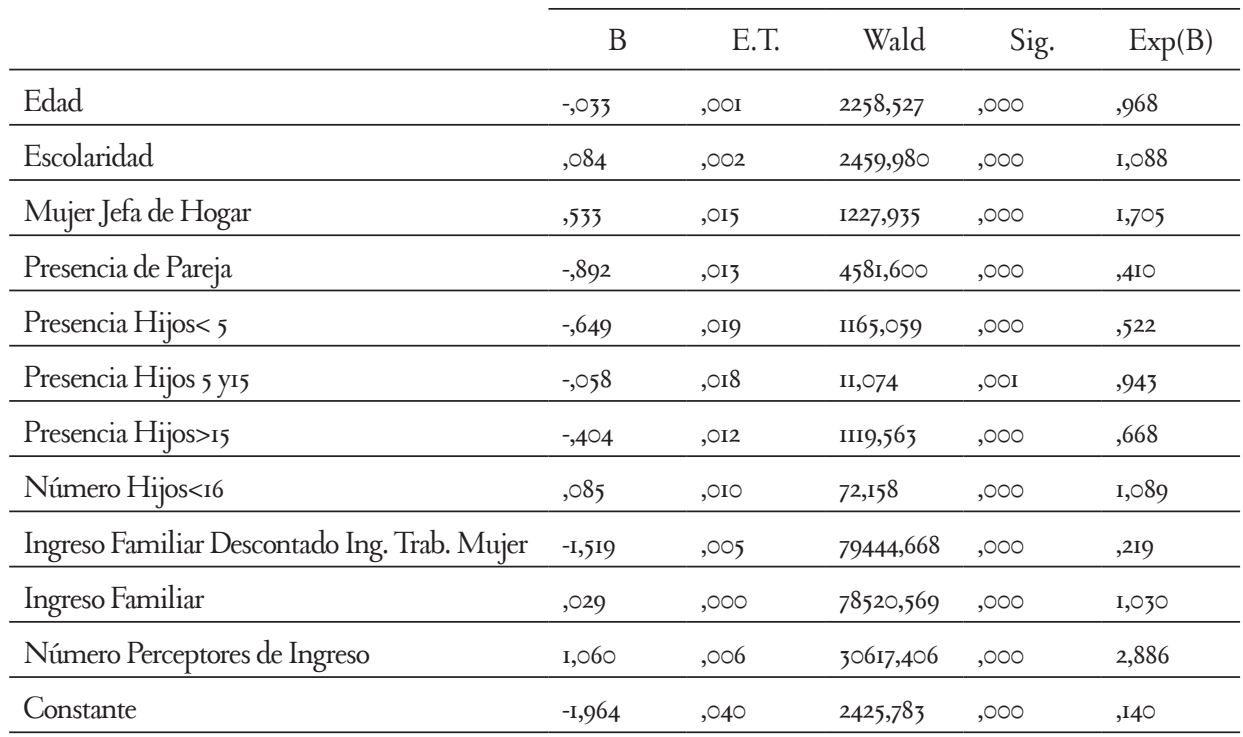

Fuente: elaboración propia 
El Cuadro 3 muestra las variables incluidas en el modelo, todas las cuales resultaron estadísticamente significativas. Son el número de perceptores de ingreso en el hogar, el número de hijos/as menores de 16 años de edad, el hecho de ser mujer jefa de hogar, tener mayor escolaridad y disponer de un mayor ingreso familiar como factores facilitadores de la inserción laboral de las mujeres rurales. Por otra parte, la presencia de hijos/as menores, en especial en edad preescolar, la tenencia de pareja y disponer de un mayor ingreso familiar no dependiente del aporte femenino, se constituyen en factores inhibidores de la participación laboral.

La modificación de las pautas de consumo en el campo, en un panorama de ingresos restrictivos por los bajos salarios, hace necesaria la incursión de más miembros del grupo familiar en el mercado del trabajo. Se busca mejorar los ingresos por la vía de aumentar el número de perceptores económicos, incentivo que se instala de manera preferencial sobre la mujer, contingente de reserva en la familia. Por cada aumento en la cantidad de perceptores de ingreso en el hogar, la probabilidad de la mujer de encontrarse empleada aumenta en 2.8 veces. El tránsito de un único perceptor económico familiar a un conjunto de sujetos generadores de ingresos da cuenta de la implementación de una estrategia de respuesta a un contexto económico poco favorable, así como a un proceso de aspiración y de movilidad que registró la estructura social rural en los últimos 20 años.

La participación laboral femenina se activa con intensidad en periodos de crisis, en familias de tamaño numeroso o de estructura monoparental. La jefatura de hogar femenina es un eficiente predictor de la participación laboral de estas. Cuando las mujeres rurales son jefas de hogar, la probabilidad de encontrarse trabajando aumenta en I.7 veces. La jefatura de hogar instala otro tipo de responsabilidades sobre ella, donde el trabajo se constituye en el principal elemento para compensar la disminución del nivel adquisitivo que implica la separación conyugal. Asimismo, el trabajo remunerado viabiliza la continuidad de proyectos de vida que se pueden reestructurar, esta vez escindidos de la pareja o marido, al reducir sus niveles de tolerancia frente a situaciones no deseadas, lo que eventualmente implica un salto cualitativo respecto de sus madres.

Otro factor que incide de modo positivo en la participación laboral femenina es el número de niños menores de i6 años de edad existentes en el hogar. Se observa que cuando aumenta esa cantidad mejoran en la mujer las probabilidades de estar empleada $(\mathrm{OR}=\mathrm{I}, 089)$, no obstante este factor está fuertemente moderado por la edad de los hijos. Cuando el número de dependientes crece de forma significativa se activa la urgencia de generar mayores recursos al aumentar las demandas sobre el grupo familiar. 
La búsqueda del equilibrio presupuestario familiar lleva al aumento del número de perceptores de ingresos y, por su intermedio, la necesidad de hacer uso de la fuerza de trabajo de reserva, principalmente constituida por mujeres dentro del espacio rural.

Esta evidencia, si bien contrasta con los hallazgos informados por otros estudios, es matizada por la edad de la prole, de forma tal que el número de hijos/as menores de 16 años como factor predictivo de la participación laboral femenina tendrá un comportamiento de intensidad diferenciada al considerar la edad de hijos/as. La presencia de hijos/as menores de 5 años de edad inhibe la participación laboral de las mujeres rurales $(\mathrm{OR}=0,522)$; por el contrario, el impacto que tiene en ellas los hijos en edad escolar, esto es entre 6 y 15 años, es mucho más atenuado y menos relevante $(\mathrm{OR}=0,943)$.

Atributos de mayor nivel de dependencia de los hijos preescolares y dificultad para delegar a terceros la protección y cuidado, estarían en la base de sustentación explicativa de la fuerza inhibidora que tiene la presencia de hijos/as pequeños en la participación laboral de la mujer. Características y condiciones que no son extensibles a los de mayor edad. Los hijos de mayor edad están escolarizados, en su gran mayoría en jornada escolar extendida y reciben incluso alimentación en su mismo centro educativo. La presencia de hijos/as ejerce presión sobre los eventuales generadores de ingresos e incentiva la inserción laboral femenina, decisión que se activará con mayor intensidad cuando los costes relativos al cuidado de los hijos no sean elevados. Los hijos en edad escolar estarían dotados de mayor independencia y gran parte de su tiempo al resguardo de un espacio institucional escolar, por lo que su presencia no necesariamente dificulta la inserción de las mujeres rurales al trabajo.

Por otra parte, los hijos en edad preescolar son significativamente más dependientes, pues precisan atención permanente, por tanto, si la madre no encuentra redes de cuidados formal (sala cuna, jardín infantil) o informal (solidaridad familiar, personal de cuidado doméstico) no tendrá la posibilidad de delegar estas responsabilidades. En el campo, la posibilidad de delegar la atención del hijo prescolar son menores, así como también son menores los niveles de renta del trabajo.

Los niveles de escolaridad $(\mathrm{OR}=1,088)$ y la posición relativa en la escala de los ingresos $(\mathrm{OR}=\mathrm{I}, 03)$ mejoran de forma relevante las probabilidades de inserción laboral de las mujeres rurales. Los años de estudio son indicador de capital humano acumulado, que el mercado del trabajo valora y que se transa a mejor precio conforme mayor sea el nivel educativo; esto aumenta, en una relación casi lineal, la 
probabilidad de estar empleada conforme aumentan los años de estudio. Los datos informan que la tasa de participación laboral de las mujeres rurales con educación media completa aumenta en I4 puntos porcentuales en comparación con las que solo tienen enseñanza básica completa (36.9\%), brecha que supera los 42 puntos porcentuales cuando se alcanza la educación superior completa (78.7\%). Si bien la escolaridad de la mujer tiene un impacto en la productividad del hogar, cuando esta es madre la productividad se incrementa en una proporción menor a los salarios provenientes del trabajo, de modo que el costo de oportunidad por unidad de bienes producidos en el hogar se ve disminuido (Legazpe, 2006).

Del mismo modo, sobre las mujeres más educadas pesan menos condicionantes de tipo sociocultural referentes a normas sociales que estigmatizan la relación laboral fuera del ámbito doméstico. Estigma social que se hace menos restrictivo conforme mejoran los niveles de renta familiar y el salario de la mujer (Goldin, 1995; Cordourier y Gómez-Galvarriato, 2004), posibilitado por el mayor capital humano acumulado. En esta situación, la presencia de pareja, factor que tiende a incidir de forma negativa en la participación laboral de las mujeres rurales, incluso se hace menos relevante.

La decisión de participar de forma laboral depende también del nivel de ingreso familiar $(\mathrm{OR}=\mathrm{I}, 03)$, pero este ingreso tendrá un comportamiento diferenciado según se considere o no el aporte económico que generan las mujeres debido a su trabajo. Se observa que conforme mayor es el nivel de ingreso familiar mayor es la participación de la mujer, pero al excluir los ingresos provenientes del trabajo de esta se suscita un efecto inverso $(\mathrm{OR}=0,219)$. El presupuesto familiar solventa las nuevas pautas de bienestar y de consumo instaladas en el campo, ingresos que en una parte significativa provienen del trabajo de sus miembros.

Si no hay transferencias, ahorros o aportes de otros miembros el sustentar estatus y consumo incentivará la participación en el mercado del trabajo, incluida la de las mujeres. La independencia económica, que se acrecienta conforme aumenta el nivel de ingreso, se sostiene de forma progresiva por el aporte pecuniario de las mujeres. Por tal razón, a las familias se les hace cada vez más difícil prescindir de este ingreso, máxime cuando estas mujeres, producto de la educación adquirida, cuentan con mayor capital humano que incrementa sus salarios y aumenta el costo de oportunidad del ocio o del tiempo dedicado a las labores domésticas.

Por otro lado, en un contexto de mayores transferencias o aporte de otros miembros del hogar al presupuesto familiar se desincentiva la participación femenina, pues en este contexto la tasa marginal de sustitución entre consumo y ocio 
tenderá a ser mayor que el salario de mercado. Cuando este ingreso no laboral de la mujer permite satisfacer determinado nivel de consumo y el salario al que se pueda aspirar en ese momento es relativamente bajo, la elección se inclinará a invertir su tiempo en las labores domésticas o incluso al estudio. Ello explicaría por qué el mayor ingreso familiar, calculado una vez excluido el aporte salarial de las mujeres, desincentiva las tasas de participación de estas. Similares hallazgos se encontrarían en recientes investigaciones realizadas en el contexto nacional chileno tales como la efectuada por Contreras, Bravo y Puentes (2005) o Encina (2008).

El contar con pareja, con independencia del tipo de vínculo, afecta negativamente la decisión de trabajar en las mujeres rurales. La existencia de un proveedor de ingreso desincentiva la decisión laboral de la mujer $(\mathrm{OR}=0,4 \mathrm{I})$, hecho que suele ser reforzado por la negativa posición actitudinal que este tiene frente al trabajo de su pareja (Méndez, 20I0), así como por las mayores responsabilidades que involucran el hogar y los hijos, situación que restringe el espacio decisional de las mujeres rurales. Aunque su impacto se ha ido atenuado con el tiempo, prueba de ello es el notorio aumento de mujeres casadas o con pareja activa en el mercado laboral, esta participación se mantiene menor a la observada en mujeres solteras o casadas con cónyuges desempleados. La disponibilidad de ingresos no laborales, esto es, no derivados del trabajo femenino, desincentiva la inserción de la cónyuge, en especial, cuando se observan diferenciales niveles educativos. En esa situación, el de mayor nivel educativo incrementa su probabilidad de participar del mercado laboral y disminuye la del otro dado el efecto renta (Castro, García y Badillo, 20II).

La edad presenta un efecto de tipo inverso sobre la participación laboral de las mujeres rurales, pues conforme aumenta la edad de estas su probabilidad de participar en el mercado laboral disminuye $(\mathrm{OR}=0,968)$. En este sentido, el grupo etario que presenta las mayores tasas de participación laboral está entre 25 y 29 años de edad (49.6\%), pues en ese rango un gran contingente de mujeres finaliza sus estudios para ingresar al mundo laboral. A partir de los 39 años comienza a disminuir la inserción laboral de las mujeres debido a que estas pertenecen a generaciones de mayor edad y por ende menos preparadas para competir desde el punto de vista laboral. Hallazgos similares han sido encontrados por Bravo, Contreras y Puentes (2005).

Por otra parte, no solo se estaría en presencia de un contingente menos atractivo para el nuevo mercado laboral femenino, vinculado de manera preponderante al área de los servicios, sino que además mujeres de mayor edad tienden a evidenciar perfiles actitudinales más conservadores y prejuiciados sobre su propia inserción laboral. Tales atributos las diferencian de las nuevas 
generaciones, más permeables y menos resistentes a las transformaciones culturales acontecidas en la sociedad, con alto impacto sobre la organización y estructura familiar y en las relaciones de género.

\section{Conclusiones}

La participación laboral de la mujer, en especial, del sector rural, constituye uno de los más significativos e importantes acontecimientos transformadores del actual mercado de trabajo chileno. Aunque con rezagos y déficit respecto de otros países del contexto latinoamericano, los datos informan de una fuerte irrupción de las mujeres rurales en la esfera laboral con tasas que en 20 Ir alcanzaba $30.8 \%$, lo que representa un aumento de un $170 \%$ respecto de 1982 .

Aunque persisten brechas laborales por zona y género que sitúan a las mujeres rurales con tasas ostensiblemente inferiores comparadas con la participación laboral femenina urbana y masculina rural, estas brechas se atenúan y reducen de forma significativa en el transcurso del tiempo. En efecto, entre 1982 y 20 I la brecha de participación laboral rural-urbana se redujo 44.I\% (25.2 pts.) entre las mujeres. Asimismo, dentro del sector rural la brecha de género se redujo $35 \%$ (30.2 pts.) para igual periodo.

Factores asociados con el perfil de una mujer trabajadora más y mejor preparada y con una favorable predisposición actitudinal hacia el trabajo; así como la existencia de un mercado laboral amplio, diversificado y flexible, estarían en la base explicativa de este fenómeno. El aumento de la participación de las mujeres rurales en el mercado laboral se ha traducido en empleabilidad efectiva, y ha mantenido estable los niveles de desempleo. Ello da cuenta de la capacidad del mercado para absorber el nuevo contingente laboral, no obstante evidenciarse una precarización de las condiciones de trabajo con una proliferación del empleo temporal y rentas reducidas.

Las mujeres rurales que se insertan en el mercado laboral se caracterizan por pertenecer a generaciones mejor preparadas, que retardan su entrada al trabajo para acumular capital humano, postergan la maternidad, retrasan la constitución de pareja y reducen la natalidad. Factores de significativa y fuerte incidencia a la hora de conseguir y mantener un trabajo remunerado, mecanismo que permite alcanzar mayores estándares de bienestar, consumo y calidad de vida en las mujeres rurales y, por su intermedio, el de sus grupos familiares.

Respecto de los factores determinantes de la participación laboral de las mujeres rurales encontramos que inciden positivamente en la probabilidad de encontrarse 
empleada: el número perceptores de ingreso familiar $(\mathrm{OR}=2,88)$; ser jefa de hogar (OR=I,70); el número de hijos/as menores de I6 años (OR=1,089); la escolaridad $(\mathrm{OR}=\mathrm{I}, 088)$; y disponer de un mayor ingreso familiar $(\mathrm{OR}=\mathrm{I}, 03)$. Por otra parte, son factores inhibidores: tener hijos/as menores, en especial en edad preescolar $(\mathrm{OR}=0,52)$; la tenencia de pareja $(\mathrm{OR}=0,4 \mathrm{I})$; $y$ disponer de un mayor ingreso familiar no dependiente del aporte femenino $(\mathrm{OR}=0,2 \mathrm{I})$

Las nuevas pautas de bienestar y consumo instaladas en el mundo rural, en un contexto de ingresos restringidos, precisan de la participación de un mayor número de perceptores, por tanto, la unidad familiar debe hacer uso de la mano de obra de reserva, constituida principalmente por mujeres mejor preparadas y con mejor perfil actitudinal hacia el trabajo. La necesidad económica se activa con mayor intensidad cuando la mujer es la única sostenedora del hogar por su calidad de mujer jefa de hogar. De igual modo, cuando los ingresos familiares no dependientes del aporte del trabajo de la mujer es mayor se inhibe la necesidad de participar por parte de esta.

Por otra parte, la presencia de hijos/as pequeños en edad preescolar y la existencia de pareja en el hogar inhiben la participación de las mujeres rurales en el mercado del trabajo, no obstante verse atenuado su efecto por el mayor capital cultural y educativo. Los hijos e hijas pequeños aumentan la demanda de manutención y cuidado sobre la madre, responsabilidad que se delega con dificultades a un tercero (institución o persona). La presencia de pareja, sea por la vía de sus aportes económicos, que hacen menos necesarios los que genera la mujer, o por las reticencias que manifiestan frente a la participación de ellas en un espacio extradoméstico ajeno a su control, así como otros elementos de carácter cultural estarían en la base explicativa de estos factores.

\section{Referencias}

Agarwal, B. (1999). Negociación y relaciones de género: dentro y fuera de la unidad doméstica. Historia Agraria, I7, 13-58.

Álvarez, G. (2002). Decisiones de fecundidad y participación laboral de la mujer en España. Investigaciones Económicas, 26(I), I87-218.

Anthopoulou, T. (2010). Rural Women in Local Agrofood Production: Between Entrepreneurial Initiatives and Family Strategies. A Case Study in Greece. Journal of Rural Studies, 26(4), 394-403. 
Attanasio, O., Low, H. y Sánchez-Marcos, V. (2004). Explaining Changes in Female Labour Supply in a Life-Cycle Model (Faculty of Economics, University of Cambridge, Working Paper 045I).

Ballara, M., Damianović, N. y Parada, S. (2010). Aporte de ingreso económico de las mujeres rurales a sus hogares. Unifem.

Becker, G. (1985). Human Capital, Effort and the Sexual Division of Labor. Journal of Labor Economics, 3(I), 33-58.

Benería, L. (1987). The Crossroads of Class and Gender: Industrial Homework, Subcontracting and Housebold Dynamics in Mexico City. Chicago: University of Chicago Press Economics Books.

Berlinsk, S., Galiani, S. y Manacorda, M. (2007 ). Giving Children a Better Start: Preschool Attendance and School-Age Profiles. Impact Evaluation, (I6), 4-30. Blau, D. y Currie, J. (2006). Preschool, Day Care and after School Care: Who's Minding the Kids? En Erik Hanushek y F. Welch (Eds.), Handbook of the Economics of Education. Amsterdam: Elsevier.

Bonvecchi, A. y Smulovitz, C. (2008). Atender necesidades, crear oportunidades o garantizar derechos. Public Disclosure Authorized, 5-8.

Bordón, P. (2006). El efecto de los jardines infantiles en la oferta laboral femenina. Análisis del caso chileno. Santiago: Universidad de Chile.

Bravo, J. y Bertranou, F. (2006). Evolución demográfica y pensiones en Chile. Santiago: OIT. Bravo, D., Contreras, D. y Puentes, E. (2005). Female Labour Force Participation in Greater Santiago, Chile: 1957-I997. A Synthetic Cohort Analysis. Journal of International Development,I7(2), I69-I86.

Cascio, E. (2006). Public Preschool and Maternal Labor Supply: Evidence from the Introduction of Kindergartens into American Public Schools. National Bureau of Economic Research.

Casen (20II). Encuesta de Caracterización Socioeconómica Nacional. Santiago: Ministerio de Desarrollo Social.

Castro, E., García, G. y Badillo, E. (20II). La participación laboral de la mujer casada y su cónyuge en Colombia: un enfoque de decisiones relacionadas. Lecturas de Economía, (74), I7I-20I.

Connelly, R. (1992). The Effect of Child Care Costs on Married Women’s Labor Force Participation. Review of Economics and Statistics, (74), 83-90.

Contreras, D. y Plaza, G. (2007). Participación laboral femenina en Chile. ¿Cuánto importan los factores culturales? Santiago: Serie Documentos de Trabajo. Departamento de Economía, Universidad de Chile. 
Contreras, D. y Plaza, G. (2010). Los factores culturales en la participación laboral de las mujeres en Chile. Feminist Economics, I6(2), 27-46.

Cordourier, G. y Gómez-Galvarriato, A. (2004). La evolución de la participación laboral de las mujeres en la industria: una visión de largo plazo. Economía Mexicana Nueva Época, I3(I), 63-104.

Cruces, G. y Galiani, S. (2007). Ferility and Female Labor Supply in Latin America: New Causal Evidence. Labour Economics, VIX(3), 565-573.

De Ferranti, D. et ál. (2005). Desigualdad en América Latina y el Caribe: ¿̇Ruptura con la historia? Washington D.C.: Banco Mundial.

Encina, J. (2008). Chile Crece Contigo: el efecto de la implementación de salas cunas públicas en participación laboral femenina,pobreza y distribución de ingresos. Santiago: PNUD.

Farah, M. y Pérez, E. (2004). Mujeres rurales y nueva ruralidad en Colombia. Bogotá D.C.: Pontificia Universidad Javeriana.

Fawaz, J. y Rodríguez, C. (2013). Mujeres rurales y trabajo en Chile central. Actitudes, factores y significaciones. Cuadernos de Desarrollo Rural, Io(72), 47-68. Fong, M. y Lokshin, M. (2000). Child Care and Women's Labor Force Participation in Romania (The World Bank, Working Paper 2400).

García, M., Glinskaya, E. y Lokshin, M. (2000). The Effect of Early Childhood Development Programs on Women's Labor Force Participation and older Children's Schooling in Kenya (The World Bank Working Paper 2376).

Gelbach, J. (2002). Public Schooling for Young Children and Maternal Labor Supply. American Economic Review, 307-322.

Goldin, C. (1995). The U-Shaped Female Labor Force Function in Economic Development and Economic History. En T. P. Schultz (Ed.) Investment in Women's Human Capital. Chicago: University of Chicago Press.

Harbart, B., Muchnik, E., Strüver, A. y Vial, I. (199I). Oferta de trabajo femenino en Santiago. Cuadernos de Economía, 28(85), 463-489.

Haugen, M. y Blekesaune, A. (2005). Farm and Off-Farm Work and Life Satisfaction among Norwegian Farm Women. Sociologia ruralis, 45(I-2), 7I-85.

Henríquez, P., Mizala, A. y Romaguera, P. (1999). Female Labor Supply in Chile. Santiago: Universidad de Chile. Centro de Economía Aplicada (CEA).

Jaumotte, F. (2003). Female Labour Force Participation: Past Trends and Main Determinants in OECD Countries. París: OECD.

Kay, C. (2008). Reflections on Latin American Rural Studies in the Neoliberal Globalization Period: A New Rurality? Development and Change, 915-943. 
Lamanna, F. y Klasen, S. (2008). The Impact of Gender Inequality in Education and Employment on Economic Growth: New Evidence for a Panel of Countries. Feminist Economics, I5(3), 91-132.

Larrañaga, O. (2006). Participación laboral de la mujer en Chile: 1958-2003. Santiago: Departamento de Economía, Universidad de Chile.

Legazpe, N. (2006). Fecundidad y participación laboral femenina: una estimación simultánea. Cuenca: Universidad de Castilla-La Mancha.

Meller, P. (2010). Carreras universitarias. Rentabilidad, selectividad y discriminación. Santiago de Chile: Uqbar.

Méndez, C. (2010). Los determinantes de la participación laboral femenina. Chile: 1996, 2001 y 2006. Santiago: Universidad de Chile.

Schkolnik, M. (2004). ¿̇Por qué es tan increiblemente baja la tasa de participación de las mujeres en Chile? Santiago: Expansiva.

Valdés, X. (2007). Notas sobre la metamorfosis de la familia en Chile. Santiago: Comisión Económica para América Latina y el Caribe, LOM Ediciones.

Weller, J. (1998). Los mercados laborales en América Latina: su evolución en el largo plazo y sus tendencias recientes. Comisión Económica para América Latina y el Caribe. 